OPEN ACCESS

Edited by:

Michel Salzet,

Lille University of Science

and Technology, France

Reviewed by:

Jonathan David Geiger,

University of North Dakota,

United States

Liubov V. Gushchina,

Nationwide Children's Hospital,

United States

*Correspondence:

Jessica L. Reynolds

jlr8@buffalo.edu

Specialty section:

This article was submitted to

Molecular Medicine,

a section of the journal

Frontiers in Cell and Developmental

Biology

Received: 02 December 2020

Accepted: 17 February 2021

Published: 01 April 2021

Citation:

Mahajan SD, Ordain NS,

Kutscher H, Karki S and Reynolds JL (2021) HIV Neuroinflammation:

The Role of Exosomes in Cell Signaling, Prognostic and Diagnostic

Biomarkers and Drug Delivery.

Front. Cell Dev. Biol. 9:637192.

doi: 10.3389/fcell.2021.637192

\section{HIV Neuroinflammation: The Role of Exosomes in Cell Signaling, Prognostic and Diagnostic Biomarkers and Drug Delivery}

\author{
Supriya D. Mahajan ${ }^{1}$, Nigel Smith Ordain ${ }^{1}$, Hilliard Kutscher ${ }^{1,2,3}$, Shanta Karki ${ }^{1}$ and \\ Jessica L. Reynolds ${ }^{1 *}$ \\ ${ }^{1}$ Department of Medicine, Jacobs School of Medicine and Biomedical Sciences, University at Buffalo, Buffalo, NY, \\ United States, ${ }^{2}$ Institute for Laser, Photonics and Biophotonics, University at Buffalo, The State University of New York, \\ Buffalo, NY, United States, ${ }^{3}$ Department of Anesthesiology, State University of New York at Buffalo, Buffalo, NY, \\ United States
}

Fifty to sixty percent of HIV-1 positive patients experience HIV-1 associated neurocognitive disorders (HAND) likely due to persistent inflammation and bloodbrain barrier (BBB) dysfunction. The role that microglia and astrocytes play in HAND pathogenesis has been well delineated; however, the role of exosomes in HIV neuroinflammation and neuropathogenesis is unclear. Exosomes are 50$150 \mathrm{~nm}$ phospholipid bilayer membrane vesicles that are responsible for cell-tocell communication, cellular signal transduction, and cellular transport. Due to their diverse intracellular content, exosomes, are well poised to provide insight into HIV neuroinflammation as well as provide for diagnostic and predictive information that will greatly enhance the development of new therapeutic interventions for neuroinflammation. Exosomes are also uniquely positioned to be vehicles to delivery therapeutics across the BBB to modulate HIV neuroinflammation. This mini-review will briefly discuss what is known about exosome signaling in the context of HIV in the central nervous system (CNS), their potential for biomarkers as well as their potential for vehicles to deliver various therapeutics to treat HIV neuroinflammation.

Keywords: HIV neuroinflammation, exosomes, signaling, delivery, blood-brain barrier

\section{INTRODUCTION}

The lifespan of patients with HIV-1 has significantly improved due to combination therapy with highly active antiretroviral therapy (HAART). However, HIV-1 associated neurocognitive disorders (HANDs) develop in $50-60 \%$ of patients and cause significant morbidity in this population (Hogan and Wilkins, 2011; Valcour et al., 2011). HAND is classified into three categories, including "asymptomatic neurocognitive impairment, mild neurocognitive disorder, or HIV-associated dementia" (Anthony and Bell, 2008; Ferrell and Giunta, 2014; Malik and Eugenin, 2016; Chivero et al., 2017; Guo and Buch, 2019; Mitra and Sharman, 2020). Confusion, headaches, cognitive motor impairment, and anxiety disorders are common and increase health-care costs (Hogan and Wilkins, 2011; Valcour et al., 2011). Central nervous system (CNS) dysfunction induced by multiple 
factors likely contributes to the progression and severity of HAND (Anthony and Bell, 2008; Ferrell and Giunta, 2014; Malik and Eugenin, 2016; Chivero et al., 2017; Guo and Buch, 2019). Microglia, CNS-resident mononuclear phagocytic cells permissible to HIV infection, are implicated in the neuropathogenesis of HIV (Schnell et al., 2001; El-Hage et al., 2006, 2015; Cherry et al., 2014; Dever et al., 2015; Joseph et al., 2015; Tang and Weidong, 2016; Sillman et al., 2018). Additionally, the largest population of cells in the CNS, astrocytes, are sources of persistent inflammation during HIV infection (Brack-Werner, 1999; Hong and Banks, 2015; Li et al., 2016; Rodriguez et al., 2017; Lutgen et al., 2020). While much is known about microglia cells and astrocytes in HIV neuroinflammation, the role that exosomes play in HIV neuropathogenesis has not been completely elucidated and is a rapidly developing field that may further contribute to the understanding of HIV neuroinflammation. This mini-review will succinctly discuss the role exosomes in HIV induced toxicity in the CNS and the potential of exosomes to deliver therapeutics to the CNS for HIV neuroinflammation.

\section{EXTRACELLULAR VESICLES}

Extracellular vesicles (EVs) are characterized as exosomes, macrovesicles, and apoptotic bodies (Ohno et al., 2013; Edgar, 2016; Fujita et al., 2018; Kalluri and LeBleu, 2020). Exosomes, in particular, are 50-150 $\mathrm{nm}$ phospholipid bilayer membrane vesicles. Exosomes are synthesized by invagination of the plasma membrane forming intracellular multivesicular bodies (MVBs), which contain intraluminal vesicles (ILVs). ILVs are then secreted as exosomes through the fusion of the MVB with the plasma membrane and exocytosis (For in-depth review on exosome biogenesis, see Edgar, 2016; Ha et al., 2016; Kalluri and LeBleu, 2020). Once released into the extracellular milieu, exosomes interact with other cells resulting in physiological changes (Ohno et al., 2013; Kalluri and LeBleu, 2020). Exosomes under normal and pathological conditions have multiple biological functions including autocrine, paracrine and endocrine communication, and immunoregulatory potential (Eldh et al., 2010). Multiple cells including but not limited to $\mathrm{B}$ and $\mathrm{T}$ lymphocytes, macrophages, astrocytes, microglia, myocytes, adipocytes, and Schwann cells produce exosomes, and exosomes are also detected in urine, serum, and saliva (Lenassi et al., 2010; Nanjundappa et al., 2011; Tang et al., 2018; Pulliam et al., 2019). Exosomes express surface markers such as LAMP1, LAMP2b, and ALIX1 proteins, and CD9, CD63, and CD81 (Edgar, 2016; Liu et al., 2019). In addition, exosomes are comprised of a variety of proteins such as heat shock proteins (HSP70, HSP90) as well as genetic materials such as microRNA (miRNA) and messenger RNA (mRNA). The content of exosomes [proteins, mRNAs, microRNAs (miRNAs), and signaling molecules] have potential impact as effectors on distant cells and tissues (Ludwig and Giebel, 2012; Ohno et al., 2013; Kalluri and LeBleu, 2020) and consequently provide a greater opportunity to identify potential biomarker molecules. Exosomes were often overlooked by researchers until findings showed that they have the potential for immunomodulatory effects (Ludwig and Giebel, 2012; Fujita et al., 2018).

\section{HIV AND EXOSOMES}

\section{Peripheral Studies}

Extensive work has been done investigating exosomes in the context of HIV in plasma and peripheral immune cells (Patters and Kumar, 2018). Overall studies have shown that exosomes facilitate the transport of viral proteins (i.e., Tat protein and Nef) and host proteins (i.e., pro- and anti-inflammatory cytokines/chemokines, markers of oxidative stress) and facilitate viral dissemination (Patters and Kumar, 2018). A few key studies are highlighted below. Studies have shown that HIV structural and accessory proteins Gag (Booth et al., 2006; Fang et al., 2007; Columba Cabezas and Federico, 2013) and Nef (Lenassi et al., 2010; Shelton et al., 2012; Aqil et al., 2013, 2014; Arenaccio et al., 2014a) are secreted in exosomes from various cells types. The HIV-1 accessory protein Nef, integral to HIV-associated immunopathogenesis, was found within plasmaderived exosomes of HIV-1-infected patients with HAND (Khan et al., 2016). Studies have shown that unspliced HIV-1 RNA sequences encoding for Gag can be incorporated in exosomes (Columba Cabezas and Federico, 2013). The exosome pathway in macrophages plays a major role in HIV budding (Nguyen et al., 2003) and facilitates viral infection of other cells (Kadiu et al., 2012). Dendritic cells transmit HIV-particles to T cells via exosomes (Wiley and Gummuluru, 2006; Izquierdo-Useros et al., 2010, 2012; Nanjundappa et al., 2011; Wang C. et al., 2013; Naslund et al., 2014). HIV-1 replication occurs in CD4 T lymphocytes exposed to exosomes derived from HIV-1-infected cells (Arenaccio et al., 2014a,b). However, other studies have shown that HIV-1 is produced independently of exosomes in CD4 + T lymphocytes (Park and He, 2010). Additionally, studies have shown that exosomes from HIV-infected patient sera contain HIV trans-activation response (TAR) element; the number of copies/ml were reduced in exosomes of HAART patients or long-term non-progressors (Narayanan et al., 2013). In macrophages, TAR RNA-exosomes also significantly increases the levels of interleukin-6 (IL-6) and tumor necrosis factor- $\beta$ (TNF- $\beta$ ) (Chen et al., 2018). These studies demonstrate the role of exosomes in modulating HIV signaling in the periphery.

\section{CNS Exosomes and HIV}

Preliminary studies have been done investigating the interactions of HIV proteins and exosomes and their subsequent effects on cells of the CNS; however, much work is still to be done to understand the role of exosomes in HIV neuroinflammation. Other studies have also examined the potential of exosomes as biomarkers for HAND. Below, a select few key studies are emphasized.

\section{Biomarkers}

Due to their diverse intracellular content, exosomes are poised to provide diagnostic and predictive information that will greatly enhance the development of new therapeutic 
interventions. Characterization of the cerebral spinal fluid (CSF) exosome proteome found that certain proteins (concentration and number) detected in exosome fractions were higher in $\mathrm{HIV}+$ subjects with HAND compared to those without HAND (see Table 1A). This study suggests that CSF exosomes may be a valuable source of biomarkers (Guha et al., 2019a), which is further supported by findings from this same laboratory that a novel CSF exosome biomarker, neurofilament light chain, correlates with neurocognitive impairment in cART-treated HIVpositive individuals (Guha et al., 2019b). A study by Sun et al. (2019) found that proteins from plasma neuron-derived enriched exosomes (NDEs) differ in HIV infection alone and cognitive impairment between men and women suggesting mechanistic sex differences (Sun et al., 2019). Potential NDE proteins that may represent biomarkers are shown in Table 1B. Combined, these studies support the potential role of exosomes for diagnostic biomarkers of HAND. Further studies are necessary to determine the possibility of exosomes as predictive biomarkers of disease progression.

\section{Exosomes and Nef}

Nef, a HIV-1 regulatory protein, stimulates viral infectivity by facilitating early events in the HIV-1 life cycle (Geyer et al., 2001; Buffalo et al., 2019). Transfecting astrocytes or microglia with Nef results in the presence of Nef in exosomes of respective cells (Raymond et al., 2016; Puzar Dominkus et al., 2017). Nef expression in human astrocytes increases the secretion of Nef-containing exosomes up to 5.5fold (Puzar Dominkus et al., 2017). Nef-containing exosomes are taken up by neurons, inducing multiple forms of stress, including oxidative, which suppresses neuron action potentials (Sami Saribas et al., 2017). Furthermore, Nef-transfected microglia-release $\mathrm{Nef}+$ exosomes that disrupt the integrity and permeability of the blood-brain barrier (BBB) (Raymond et al., 2016). The expression of the BBB tight junction protein, ZO-1, decreases following exposure to microglia-derived exosomes containing Nef. Microglia exposed to exosomes containing Nef had increases in the levels of IL-12, IL-8, IL6, RANTES, and IL-17A (Raymond et al., 2016). Interestingly, in neuroblastoma cells, Nef protein within plasma exosomes induces production and secretion of amyloid beta protein. This study demonstrates the role that peripheral exosomes may play in neuroinflammation or HAND, as amyloid beta $(A \beta)$ production may increase the severity of HAND (Khan et al., 2016). Elegant studies from Michal Toborek's laboratory demonstrate that HIV infection accelerates the release of brain endothelial exosomes and alters exosome-A $\beta$ levels. These exosomes transfer $\mathrm{A} \beta$ to astrocytes (András et al., 2017).

TABLE 1 | (A) Potential Biomarkers of HAND; (B) potential biomarkers in males and females with mild impairment.

\begin{tabular}{|c|c|c|}
\hline \multicolumn{3}{|c|}{ A. Potential biomarkers of HAND } \\
\hline Category/function & Proteins & Citations \\
\hline \multicolumn{3}{|c|}{ Example of some proteins in exosome fractions that were higher in HIV + subjects with HAND compared to those without HAND } \\
\hline Inflammatory/immune response & $\begin{array}{l}\text { Annexins, C-reactive protein, enolase, human } \\
\text { leukocyte antigen }(H\llcorner A) \text {, }\end{array}$ & Guha et al., 2019a \\
\hline Stress response proteins & $\begin{array}{l}\text { Parkinsonism associated deglycase } \\
\text { Peroxiredoxins, alpha-synuclein, synuclein, } \\
\text { vimentins }\end{array}$ & Guha et al., 2019a \\
\hline Neuronal & $\begin{array}{l}\text { Cell adhesion, ankyrin-binding protein, } \\
\text { Neuroplastin, Neurexins }\end{array}$ & Guha et al., 2019a \\
\hline Astrocyte proteins & $\begin{array}{l}\text { Aldehyde dehydrogenase, glial fibrillary acidic } \\
\text { protein, glutamine synthetase, calcium-binding } \\
\text { peptide }\end{array}$ & Guha et al., 2019a \\
\hline Choroid plexus protein & $\begin{array}{l}\text { ATPase } \mathrm{Na}+/ \mathrm{K}+\text { transporting subunit alpha } \\
2 \text {, ATP synthase, } \mathrm{H}+\text { transporting, } \\
\text { mitochondrial F1 complex, beta polypeptide }\end{array}$ & Guha et al., 2019a \\
\hline
\end{tabular}

B. Potential biomarkers in males and females with mild impairment

\begin{tabular}{|c|c|c|}
\hline Category/function & Proteins & Citations \\
\hline \multicolumn{3}{|c|}{ Example of proteins from NDE from women with mild impairment that were significantly decreased } \\
\hline Lysosomal cysteine protease & Cathepsin S & Sun et al., 2019 \\
\hline Microtubule-associated protein & Total tau & Sun et al., 2019 \\
\hline Cell recognition and cell-cell adhesion & Neuronal cell adhesion molecule & Sun et al., 2019 \\
\hline Cell adhesion molecule & Contactin-5 & Sun et al., 2019 \\
\hline \multicolumn{3}{|c|}{ Example of proteins from NDE that were increased from cognitively impaired men } \\
\hline Blood coagulation/fibrinolysis, inflammation & Carboxypeptidase M & Sun et al., 2019 \\
\hline Calcium-dependent cell adhesion proteins & Cadherin 3 & Sun et al., 2019 \\
\hline Endoplasmic reticulum (ER) stress-inducible neurotrophic factor. & $\begin{array}{l}\text { Mesencephalic astrocyte-derived neurotropic } \\
\text { factor }\end{array}$ & Sun et al., 2019 \\
\hline
\end{tabular}


They have also found that HIV along with $\mathrm{A} \beta$ alter brain endothelial exosomes proteome, which disrupts networks and functional interactions (András et al., 2017, 2020). Overall, this body of work demonstrates the potential contribution of Nefcontaining exosomes in the development of neuroinflammation associated with HIV.

\section{Exosomes and Tat}

HIV Trans-activating regulatory protein (Tat) stimulates transcription from the viral long terminal repeat promoter (Das et al., 2011). Buch's laboratory has done pioneering work in Tat-induced expression of microRNAs (miRNA) in exosomes. miRNAs are non-coding RNAs of 19-22 nucleotides that regulate gene expression and cell homeostasis (Hamrick et al., 2010; Smith-Vikos and Slack, 2012; Kim et al., 2014). HIV Tat increases the release of exosomes containing miR-29b (Hu et al., 2012) in astrocytes. Neuronal cells treated with these exosomes had decreases in neurotrophic factor platelet-derived growth factor expression, as well as a decrease in viability of neurons (Hu et al., 2012). Furthermore, Tat-stimulated astrocytes release miR-9 in exosomes that are engulfed by microglia. This prompts a change in microglia migratory phenotype (Yang et al., 2018). In astrocytes and neurons, HIV-1 Tat protein significantly upregulates the number of exosomes containing miR-132. Tat-expressing astrocytes release exosomes with miR-132 that are taken up by neurons, inducing neurite shortening and increases in neurotoxicity (Rahimian and He, 2016). Engineered exosomal Tat adeno-associated viruses significantly reduce the expression levels of synaptophysin (synaptic marker) in the brain of mice, demonstrating HIV-1 Tat protein attributes to synaptic damage (Tang et al., 2020). Exosomes from CSF samples were positive for Tat protein and retained transactivation activity, indicating that Tat present in CSF is functional and likely found in exosomes, further implicating the role of exosomal Tat in neurotoxicity (Henderson et al., 2019). Cumulatively, these data provide new mechanistic insights in Tat proteins signaling and neurotoxicity and demonstrate the potential role of exosomes in HIV signaling both in HIV-related neuroinflammation and neurotoxicity. Additional studies are necessary to further demarcate the role of exosomes in Tat function, transport, and association with HAND.

\section{Delivery of Therapeutics to the CNS Using Exosomes}

Targeting and delivering therapeutics to the CNS has been a longstanding challenge in biomedical research. The BBB separates circulating blood from the brain extracellular fluid. It is a highly selective permeable barrier that regulates diffusion of molecules into the brain (Serlin et al., 2015; Reynolds and Mahato, 2017; Reynolds and Mahajan, 2020). Only small, lipophilic compounds $(<400-500 \mathrm{Da})$ or hydrophobic molecules (e.g., $\mathrm{O}_{2}, \mathrm{CO}_{2}$, hormones) can cross the $\mathrm{BBB}$ (Reynolds and Mahato, 2017; Reynolds and Mahajan, 2020). The BBB contains endogenous, receptor-mediated, and efflux transporters. Endogenous transporters mediate selective uptake of watersoluble nutrients (e.g., glucose and amino acids), whereas, receptor-mediated transporters mediate the transport of large molecules [e.g., insulin and transferrin (Tf)]. Furthermore, efflux transporters [e.g., P-glycoprotein (Pgp)] pump substrates out of the brain (Abbott, 2004, 2005; Reynolds and Mahato, 2017; Reynolds and Mahajan, 2020). Multiple research efforts have been devoted to develop ways to increase the passage of therapeutics across the $\mathrm{BBB}$ and to increase the bioavailability of drugs in the CNS. Exosomes have characteristics of effective drugdelivery vehicles (van Dommelen et al., 2012; Lai et al., 2013; Aryani and Denecke, 2016; Ha et al., 2016). The structure of exosomes is a lipid bilayer membrane that allows for drug loading. The exosome lipid membrane surrounds a hydrophilic core (Frydrychowicz et al., 2015; Luan et al., 2017). Therapeutic agents are incorporated into exosomes through active or passive encapsulation (Luan et al., 2017). Diverse therapeutic agents have been loaded in exosomes such as small interfering RNA (siRNA) or small molecules (Alvarez-Erviti et al., 2011; El Andaloussi et al., 2013; Wang C. et al., 2019; Wang H. et al., 2019; Reynolds and Mahajan, 2020). Exosomes are slightly negatively charged improving their stability in blood circulation, and their size helps to eliminate renal clearance (Luan et al., 2017). They also readily diffuse across plasma membranes (Chen et al., 2016; Matsumoto et al., 2017). Multiple studies have used exosomes to deliver therapeutics to the brain for multiple conditions such as stroke (Li et al., 2020; Venkat et al., 2020), and traumatic brain injury (Chen et al., 2020). Using a zebrafish model, Yang et al. (2015) show that exosomes deliver paclitaxel and doxorubicin (cancer therapeutics) across the BBB. There is a broad potential for exosomes vehicles for the treatment of multiple CNS diseases, with multiple studies previously published but too numerous to discuss, with a few studies are highlighted in Table 2. One of the first studies to demonstrate the use of exosomes to deliver siRNA to the CNS was by Alvarez-Erviti et al. (2011). They demonstrated RVG-targeted exosomes delivered GAPDH siRNA to neurons, microglia, and oligodendrocytes in the brain, inducing in gene knockdown (Alvarez-Erviti et al., 2011). In regards to HIV, Tang et al. (2018) loaded exosomes with HIV Tat protein and these exosomes reactivated primary HIV-infected CD4 + T-cells (Tang et al., 2018). Our laboratory has used microglia-derived exosomes to deliver siRNA across a human BBB model that contains

TABLE 2 | Select examples of drug delivery to the CNS.

\begin{tabular}{lcc}
\hline Source of exosomes & Therapeutic & Citations \\
\hline $\begin{array}{l}\text { Stroke } \\
\text { Bone marrow mesenchymal stem cells } \\
\text { miR-133b+ mesenchymal stem cells }\end{array}$ & miR-124 & Yang et al., 2017 \\
Alzheimer's disease & & Xin et al., 2013 \\
$\begin{array}{l}\text { Plasma } \\
\text { Dendritic cell }\end{array}$ & Quercetin & Qi et al., 2020 \\
$\begin{array}{l}\text { Parkinson's disease } \\
\text { Macrophage }\end{array}$ & GADH siRNA & Alvarez-Erviti et al., 2011 \\
$\begin{array}{l}\text { Blood } \\
\text { Glioblastoma }\end{array}$ & \\
U-87 & Dopatalase & Haney et al., 2015 \\
Glioblastoma cells & Quclitaxel & Salarpour et al., 2019 \\
& miR-21 & Kim et al., 2020
\end{tabular}


HIV latently infected human telomerase reverse transcriptase immortalized human microglial cells (HTHU-HIV) on the brain side of the model. This study was done to demonstrate a proof of concept that exosomes can transmigrate through the BBB model (Figure 1). We applied microglia-derived exosomes loaded with siRNA specific to Tspan 2 to the apical side of the BBB (Reynolds and Mahajan, 2020). Tspan2 siRNA decreases Tspan2 gene expression in HTHU-HIV microglia at the basolateral (brain) side of the BBB model. Knockout of Tspan2 directly led to a decrease in C-X-C motif chemokine 12 (CXCL12) and C-X-C chemokine receptor type 4 (CXCR4) gene expression in HTHUHIV microglia. The inflammatory response of HTHU latently infected microglia cells was also altered as the gene expression levels of the IL-13 and IL-10 decreases, and gene expression levels for the Fc gamma receptor 2A(FCGR2A) and TNF- $\alpha$ increase (Reynolds and Mahajan, 2020). Overall, this study demonstrates a proof of concept that brain-derived exosomes can be used to cross the BBB to deliver RNA therapeutics to modify genes and proteins that are associated with HIV neuroinflammation. Qiu et al. (2018) elegantly discuss the potential of exosomes to deliver anti-HIV RNA-loaded exosomes (see review Qiu et al., 2018). The potential to use exosomes to delivery HIV therapeutics to the brain is an untapped field and has the potential to deliver therapeutics that reduce inflammatory mediators (inhibitors, siRNA), reduce viral replication (delivery of ARVs), deliver miRNA. However, there are some limitations to using exosomes as drug-delivery vehicles; these limitations are slowly being resolved as the field advances. The heterogenous nature of the content of exosomes, including proteins, lipids, miRNAs, RNA, and DNA, is a primary concern (Record et al., 2011; Ludwig and Giebel, 2012). Loading exosomes from one cell type with a therapeutic and delivering to another cell type is likely to deliver the contents to the latently infected cell type. As described above, miRNA found in exosomes impacts target cells (Hu et al., 2012; Yang et al., 2018). A major limitation of our study described above was in addition to delivering Tspan2 siRNA to microglia cells (Luan et al., 2017; Reynolds and Mahajan, 2020); we likely delivered other contents in exosomes such as DNA, protein, or miRNA. However, we isolated exosomes from microglia cells so we were likely delivering similar contents to the lately infected microglia cells (Reynolds and Mahajan, 2020). One mechanism to overcome this limitation is to use "synthetic exosomes," such as liposomes or nanoparticles. For example, liposomes conjugated to cell-penetrating peptides increased transfection efficiency of encapsulated plasmid DNA delivery across the BBB (Dos Santos Rodrigues et al., 2019). Our laboratory has shown that Tfconjugated quantum rods deliver Saquinavir across the BBB to increase CNS concentrations (Mahajan et al., 2010). An excellent review of nanoparticles for delivery of therapeutics for CNS infection nicely describes the potential nanoparticles (please see review DeMarino et al., 2017). An extensive discussion of nanoparticles is beyond the scope of this minireview. Another

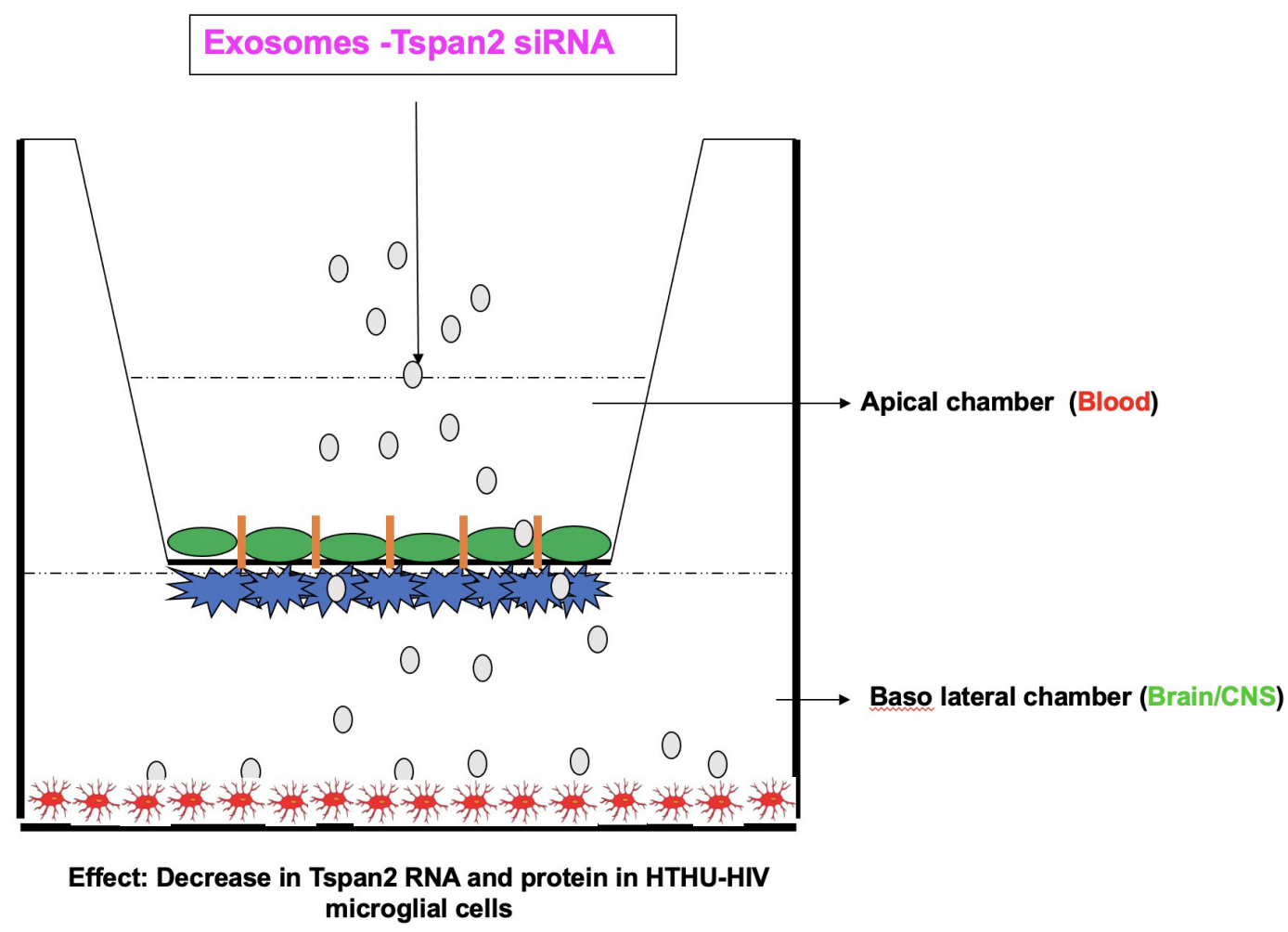

FIGURE 1 | Blood-brain barrier model demonstrating proof of concept of exosome delivery of Tspan2 across the BBB to latently infected microglia on the brain side. Schematic adapted from Reynolds and Mahajan (2020). 
limitation, in particular with HIV, is the size of viral particles that are similarly sized to the exosome. While isolating exosomes from HIV-infected cells, plasma, serum, or CSF, one may also be concentrating HIV virions. However, Kashanchi's laboratory (Narayanan et al., 2013; Jaworski et al., 2014; DeMarino et al., 2017; Henderson et al., 2019), a pioneer laboratory in HIV and exosomes, has developed a technique that allows for separation of exosomes from HIV virions. An additional problem with using exosomes as delivery is specific cell targeting. Isolating exosomes from plasma or serum to be used for drug delivery across the BBB does not allow for specific cell type (i.e., astrocytes, microglia) targeting, which is especially important in CNS disease. Several studies have transfected cells to produce exosomes with targeting proteins on their surface or during isolation have enriched for certain proteins (Alvarez-Erviti et al., 2011; El Andaloussi et al., 2013; Sun et al., 2019). Nevertheless, the study of exosomes as drug-delivery vehicles is an exciting field.

\section{DISCUSSION}

The field of EVs, including exosomes, is a rapidly advancing with significant potential for determining the underpinnings of HIV cellular signaling, transport, and treatment of HAND. Understanding the role that exosomes play in CNS and BBB

\section{REFERENCES}

Abbott, N. J. (2004). Prediction of blood-brain barrier permeation in drug discovery from in vivo, in vitro and in silico models. Drug Discov. Today Technol. 1, 407-415. doi: 10.1016/j.ddtec.2004.11.014

Abbott, N. J. (2005). Dynamics of CNS barriers: evolution, differentiation, and modulation. Cell. Mol. Neurobiol. 25, 5-23. doi: 10.1007/s10571-004-1374-y

Alvarez-Erviti, L., Seow, Y., Yin, H., Betts, C., Lakhal, S., and Wood, M. (2011). Delivery of siRNA to the mouse brain by systemic injection of targeted exosomes. Nat. Biotechnol. 29, 341-345. doi: 10.1038/nbt. 1807

András, I. E., Leda, A., Contreras, M. G., Bertrand, L., Park, M., Skowronska, M., et al. (2017). Extracellular vesicles of the blood-brain barrier: role in the HIV-1 associated amyloid beta pathology. Mol. Cell. Neurosci. 79, 12-22. doi: 10.1016/j.mcn.2016.12.006

András, I. E., Sewell, B. B., and Toborek, M. (2020). HIV-1 and amyloid beta remodel proteome of brain endothelial extracellular vesicles. Int. J. Mol. Sci. 21:2741. doi: 10.3390/ijms21082741

Anthony, I. C., and Bell, J. E. (2008). The neuropathology of HIV/AIDS. Int. Rev. Psychiatry 20, 15-24. doi: 10.1080/09540260701862037

Aqil, M., Naqvi, A. R., Bano, A. S., and Jameel, S. (2013). The HIV-1 Nef protein binds argonaute- 2 and functions as a viral suppressor of RNA interference. PLoS One 8:e74472. doi: 10.1371/journal.pone.0074472

Aqil, M., Naqvi, A. R., Mallik, S., Bandyopadhyay, S., Maulik, U., and Jameel, S. (2014). The HIV Nef protein modulates cellular and exosomal miRNA profiles in human monocytic cells. J. Extracell. Vesicles 3:10.3402/jev.v3.23129.

Arenaccio, C., Chiozzini, C., Columba-Cabezas, S., Manfredi, F., Affabris, E., Baur, A., et al. (2014a). Exosomes from human immunodeficiency virus type 1 (HIV1)-infected cells license quiescent CD4+ T lymphocytes to replicate HIV-1 through a Nef- and ADAM17-dependent mechanism. J. Virol. 88, 11529-11539. doi: 10.1128/jvi.01712-14

Arenaccio, C., Chiozzini, C., Columba-Cabezas, S., Manfredi, F., and Federico, M. (2014b). Cell activation and HIV-1 replication in unstimulated CD4+ $\mathrm{T}$ lymphocytes ingesting exosomes from cells expressing defective HIV-1. Retrovirology 11:46. doi: 10.1186/1742-4690-11-46 dysfunction, and neurotoxicity in the CNS that contributes to the progression of HAND, will allow for the development of better treatment modalities, delivery vehicles, and potential biomarkers. Due to their diverse intracellular content, exosomes are poised to provide diagnostic and predictive information that will greatly enhance the development of new therapeutic interventions for neuroinflammation.

\section{AUTHOR CONTRIBUTIONS}

SM, SK, and JR: concept and writing. NO and HK: writing. All authors contributed to the article and approved the submitted version.

\section{FUNDING}

This work was supported by the 1R01AI129649 (NIAID) (JR), 1R01DA047410 (NIDA) (SM), and the National Center for Advancing Translational Sciences of the National Institutes of Health under award numbers UL1TR001412 and KL2TR001413 (JR). The content was solely the responsibility of the authors and does not necessarily represent the official views of the NIH.

Aryani, A., and Denecke, B. (2016). Exosomes as a nanodelivery system: a key to the future of neuromedicine? Mol. Neurobiol. 53, 818-834. doi: 10.1007/s12035014-9054-5

Booth, A. M., Fang, Y., Fallon, J. K., Yang, J. M., Hildreth, J. E., and Gould, S. J. (2006). Exosomes and HIV Gag bud from endosome-like domains of the T cell plasma membrane. J. Cell Biol. 172, 923-935. doi: 10.1083/jcb.200508014

Brack-Werner, R. (1999). Astrocytes: HIV cellular reservoirs and important participants in neuropathogenesis. Aids 13, 1-22. doi: 10.1097/00002030199901140-00003

Buffalo, C. Z., Iwamoto, Y., Hurley, J. H., and Ren, X. (2019). How HIV Nef proteins hijack membrane traffic to promote infection. J. Virol. 93:e01322-19.

Chen, C. C., Liu, L., Ma, F., Wong, C. W., Guo, X. E., Chacko, J. V., et al. (2016). Elucidation of exosome migration across the blood-brain barrier model in vitro. Cell. Mol. Bioeng. 9, 509-529. doi: 10.1007/s12195-016-0458-3

Chen, L., Feng, Z., Yue, H., Bazdar, D., Mbonye, U., Zender, C., et al. (2018). Exosomes derived from HIV-1-infected cells promote growth and progression of cancer via HIV TAR RNA. Nat. Commun. 9:4585.

Chen, Y., Li, J., Ma, B., Li, N., Wang, S., Sun, Z., et al. (2020). MSCderived exosomes promote recovery from traumatic brain injury via microglia/macrophages in rat. Aging (Albany NY) 12, 18274-18296. doi: 10. 18632/aging.103692

Cherry, J., Olschowka, J., and O'banion, M. K. (2014). Neuroinflammation and M2 microglia: the good, the bad, and the inflamed. J. Neuroinflammation 11:98. doi: 10.1186/1742-2094-11-98

Chivero, E. T., Guo, M. L., Periyasamy, P., Liao, K., Callen, S. E., and Buch, S. (2017). HIV-1 Tat primes and activates microglial NLRP3 inflammasomemediated neuroinflammation. J. Neurosci. 37, 3599-3609. doi: 10.1523/ jneurosci.3045-16.2017

Columba Cabezas, S., and Federico, M. (2013). Sequences within RNA coding for HIV-1 Gag p17 are efficiently targeted to exosomes. Cell Microbiol. 15, 412-429. doi: $10.1111 / \mathrm{cmi} .12046$

Das, A. T., Harwig, A., and Berkhout, B. (2011). The HIV-1 Tat protein has a versatile role in activating viral transcription. J. Virol. 85, 9506-9516. doi: 10.1128/jvi.00650-11

DeMarino, C., Schwab, A., Pleet, M., Mathiesen, A., Friedman, J., El-Hage, N., et al. (2017). Biodegradable nanoparticles for delivery of therapeutics in CNS 
infection. J. Neuroimmune Pharmacol. 12, 31-50. doi: 10.1007/s11481-0169692-7

Dever, S. M., Rodriguez, M., Lapierre, J., Costin, B. N., and El-Hage, N. (2015). Differing roles of autophagy in HIV-associated neurocognitive impairment and encephalitis with implications for morphine co-exposure. Front. Microbiol. 6:653.

Dos Santos Rodrigues, B., Lakkadwala, S., Kanekiyo, T., and Singh, J. (2019). Development and screening of brain-targeted lipid-based nanoparticles with enhanced cell penetration and gene delivery properties. Int. J. Nanomed. 14, 6497-6517. doi: 10.2147/ijn.s215941

Edgar, J. R. (2016). Q\&A: what are exosomes, exactly? BMC Biol. 14:46.

El Andaloussi, S., Lakhal, S., Mager, I., and Wood, M. J. (2013). Exosomes for targeted siRNA delivery across biological barriers. Adv. Drug Deliv. Rev. 65, 391-397. doi: 10.1016/j.addr.2012.08.008

Eldh, M., Ekström, K., Valadi, H., Shostrand, M., Olsoon, B., Jernas, M., et al. (2010). Exosomes communicate protective messages during oxidative stress; possible role of exosomal shuttle RNA. PLoS One 5:e15353. doi: 10.1371/ journal.pone.0015353

El-Hage, N., Rodriguez, M., Dever, S. M., Masvekar, R. R., Gewirtz, D. A., and Shacka, J. J. (2015). HIV-1 and morphine regulation of autophagy in microglia: limited interactions in the context of HIV-1 infection and opioid abuse. J. Virol. 89, 1024-1035. doi: 10.1128/jvi.02022-14

El-Hage, N., Wu, G., Wang, J., Ambati, J., Knapp, P. E., Reed, J. L., et al. (2006). HIV-1 Tat and opiate-induced changes in astrocytes promote chemotaxis of microglia through the expression of MCP-1 and alternative chemokines. Glia 53, 132-146. doi: 10.1002/glia.20262

Fang, Y., Wu, N., Gan, X., Yan, W., Morrell, J. C., and Gould, S. J. (2007). Higher-order oligomerization targets plasma membrane proteins and HIV gag to exosomes. PLoS Biol. 5:e158. doi: 10.1371/journal.pbio.0050158

Ferrell, D., and Giunta, B. (2014). The impact of HIV-1 on neurogenesis: implications for HAND. Cell Mol. Life Sci. 71, 4387-4392. doi: 10.1007/s00018014-1702-4

Frydrychowicz, M., Kolecka-Bednarczyk, A., Madejczyk, M., Yasar, S., and Dworacki, G. (2015). Exosomes - structure, biogenesis and biological role in non-small-cell lung cancer. Scand. J. Immunol. 81, 2-10. doi: 10.1111/sji.12247

Fujita, Y., Kadota, T., Araya, J., Ochiya, T., and Kuwano, K. (2018). Extracellular vesicles: new players in lung immunity. Am. J. Respir. Cell. Mol. Biol. 58, 560-565. doi: 10.1165/rcmb.2017-0293tr

Geyer, M., Fackler, O. T., and Peterlin, B. M. (2001). Structure-function relationships in HIV-1 Nef. EMBO Rep. 2, 580-585. doi: 10.1093/emboreports/kve141

Guha, D., Lorenz, D. R., Misra, V., Chettimada, S., Morgello, S., and Gabuzda, D. (2019a). Proteomic analysis of cerebrospinal fluid extracellular vesicles reveals synaptic injury, inflammation, and stress response markers in HIV patients with cognitive impairment. J. Neuroinflammation 16:254.

Guha, D., Mukerji, S. S., Chettimada, S., Misra, V., Lorenz, D. R., Morgello, S., et al. (2019b). Cerebrospinal fluid extracellular vesicles and neurofilament light protein as biomarkers of central nervous system injury in HIV-infected patients on antiretroviral therapy. Aids 33, 615-625. doi: 10.1097/qad. 0000000000002121

Guo, M. L., and Buch, S. (2019). Neuroinflammation \& pre-mature aging in the context of chronic HIV infection and drug abuse: role of dysregulated autophagy. Brain Res. 1724:146446.

Ha, D., Yang, N., and Nadithe, V. (2016). Exosomes as therapeutic drug carriers and delivery vehicles across biological membranes: current perspectives and future challenges. Acta Pharm. Sin. B 6, 287-296. doi: 10.1016/j.apsb.2016.02.001

Hamrick, M. W., Herberg, S., Arounleut, P., He, H. Z., Shiver, A., Qi, R. Q., et al. (2010). The adipokine leptin increases skeletal muscle mass and significantly alters skeletal muscle miRNA expression profile in aged mice. Biochem. Biophys. Res. Commun. 400, 379-383. doi: 10.1016/j.bbrc.2010.08.079

Haney, M. J., Klyachko, N. L., Zhao, Y., Gupta, R., Plotnikova, E. G., He, Z., et al. (2015). Exosomes as drug delivery vehicles for Parkinson's disease therapy. J. Control Release 207, 18-30.

Henderson, L. J., Johnson, T. P., Smith, B. R., Reoma, L. B., Santamaria, U. A., Bachani, M., et al. (2019). Presence of Tat and transactivation response element in spinal fluid despite antiretroviral therapy. Aids 33(Suppl. 2), S145-S157.

Hogan, C., and Wilkins, E. (2011). Neurological complications in HIV. Clin. Med. $11,571-575$.
Hong, S., and Banks, W. A. (2015). Role of the immune system in HIV-associated neuroinflammation and neurocognitive implications. Brain Behav. Immun. 45, 1-12. doi: 10.1016/j.bbi.2014.10.008

Hu, G., Yao, H., Chaudhuri, A. D., Duan, M., Yelamanchili, S. V., Wen, H., et al. (2012). Exosome-mediated shuttling of microRNA-29 regulates HIV Tat and morphine-mediated neuronal dysfunction. Cell Death Dis. 3:e381. doi: 10.1038/ cddis.2012.114

Izquierdo-Useros, N., Lorizate, M., Puertas, M. C., Rodriguez-Plata, M. T., Zangger, N., Erikson, E., et al. (2012). Siglec-1 is a novel dendritic cell receptor that mediates HIV-1 trans-infection through recognition of viral membrane gangliosides. PLoS Biol. 10:e1001448. doi: 10.1371/journal.pbio. 1001448

Izquierdo-Useros, N., Naranjo-Gomez, M., Erkizia, I., Puertas, M. C., Borras, F. E., Blanco, J., et al. (2010). HIV and mature dendritic cells: trojan exosomes riding the Trojan horse? PLoS Pathog. 6:e1000740. doi: 10.1371/journal.ppat.1000740

Jaworski, E., Saifuddin, M., Sampey, G., Shafagati, N., Van Duyne, R., Iordanskiy, S., et al. (2014). The use of Nanotrap particles technology in capturing HIV-1 virions and viral proteins from infected cells. PLoS One 9:e96778. doi: 10.1371/ journal.pone.0096778

Joseph, S. B., Arrildt, K. T., Sturdevant, C. B., and Swanstrom, R. (2015). HIV-1 target cells in the CNS. J. Neurovirol. 21, 276-289. doi: 10.1007/s13365-0140287- $\mathrm{x}$

Kadiu, I., Narayanasamy, P., Dash, P. K., Zhang, W., and Gendelman, H. E. (2012). Biochemical and biologic characterization of exosomes and microvesicles as facilitators of HIV-1 infection in macrophages. J. Immunol. 189, 744-754. doi: 10.4049/jimmunol.1102244

Kalluri, R., and LeBleu, V. S. (2020). The biology, function, and biomedical applications of exosomes. Science 367:eaau6977. doi: 10.1126/science.aau6977

Khan, M. B., Lang, M. J., Huang, M. B., Raymond, A., Bond, V. C., Shiramizu, B., et al. (2016). Nef exosomes isolated from the plasma of individuals with HIV-associated dementia (HAD) can induce Abeta(1-42) secretion in SH-SY5Y neural cells. J. Neurovirol. 22, 179-190. doi: 10.1007/s13365-0150383-6

Kim, G., Kim, M., Lee, Y., Byun, J. W., Hwang, D. W., and Lee, M. (2020). Systemic delivery of microRNA-21 antisense oligonucleotides to the brain using T7-peptide decorated exosomes. J. Control Release 317, 273-281.

Kim, J. Y., Park, Y. K., Lee, K. P., Lee, S. M., Kang, T. W., Kim, H. J., et al. (2014). Genome-wide profiling of the microRNA-mRNA regulatory network in skeletal muscle with aging. Aging 6, 524-544. doi: 10.18632/aging.100677

Lai, R. C., Yeo, R. W., Tan, K. H., and Lim, S. K. (2013). Exosomes for drug delivery - a novel application for the mesenchymal stem cell. Biotechnol. Adv. 31, 543-551. doi: 10.1016/j.biotechadv.2012.08.008

Lenassi, M., Cagney, G., Liao, M., Vaupotic, T., Bartholomeeusen, K., Cheng, Y., et al. (2010). HIV Nef is secreted in exosomes and triggers apoptosis in bystander CD4+ T cells. Traffic 11, 110-122. doi: 10.1111/j.1600-0854.2009. 01006.x

Li, F., Zhao, L., Shi, Y., and Liang, J. (2020). Edaravone-loaded macrophage-derived exosomes enhance neuroprotection in the rat permanent middle cerebral artery occlusion model of stroke. Mol. Pharm. 17, 3192-3201. doi: 10.1021/acs. molpharmaceut.0c00245

Li, G. H., Henderson, L., and Nath, A. (2016). Astrocytes as an HIV reservoir: mechanism of HIV Infection. Curr. HIV Res. 14, 373-381. doi: 10.2174/ $1570162 \times 14666161006121455$

Liu, W., Bai, X., Zhang, A., Huang, J., Xu, S., and Zhang, J. (2019). Role of exosomes in central nervous system diseases. Front. Mol. Neurosci. 12:240.

Luan, X., Sansanaphongpricha, K., Myers, I., Chen, H., Yuan, H., and Sun, D. (2017). Engineering exosomes as refined biological nanoplatforms for drug delivery. Acta Pharmacol. Sin. 38, 754-763. doi: 10.1038/aps.2017.12

Ludwig, A. K., and Giebel, B. (2012). Exosomes: small vesicles participating in intercellular communication. Int. J. Biochem. Cell. Biol. 44, 11-15. doi: 10.1016/ j.biocel.2011.10.005

Lutgen, V., Narasipura, S. D., Barbian, H. J., Richards, M., Wallace, J., Razmpour, R., et al. (2020). HIV infects astrocytes in vivo and egresses from the brain to the periphery. PLoS Pathog. 16:e1008381. doi: 10.1371/journal.ppat.1008381

Mahajan, S. D., Roy, I., Xu, G., Yong, K. T., Ding, H., Aalinkeel, R., et al. (2010). Enhancing the delivery of anti retroviral drug "Saquinavir" across the blood brain barrier using nanoparticles. Curr. HIV Res. 8, 396-404. doi: 10.2174/ 157016210791330356 
Malik, S., and Eugenin, E. A. (2016). Mechanisms of HIV neuropathogenesis: role of cellular communication systems. Curr. HIV Res. 14, 400-411. doi: 10.2174/1570162x14666160324124558

Matsumoto, J., Stewart, T., Sheng, L., Li, N., Bullock, K., Song, N., et al. (2017). Transmission of alpha-synuclein-containing erythrocyte-derived extracellular vesicles across the blood-brain barrier via adsorptive mediated transcytosis: another mechanism for initiation and progression of Parkinson's disease? Acta Neuropathol. Commun. 5, 71-71-16.

Mitra, P., and Sharman, T. (2020). "HIV neurocognitive disorders," in StatPearls (Treasure Island, FL: StatPearls Publishing).

Nanjundappa, R. H., Wang, R., Xie, Y., Umeshappa, C. S., Chibbar, R., Wei, Y., et al. (2011). GP120-specific exosome-targeted T cell-based vaccine capable of stimulating DC- and CD4(+) T-independent CTL responses. Vaccine 29, 3538-3547. doi: 10.1016/j.vaccine.2011.02.095

Narayanan, A., Iordanskiy, S., Das, R., Van Duyne, R., Santos, S., Jaworski, E., et al. (2013). Exosomes derived from HIV-1-infected cells contain trans-activation response element RNA. J. Biol. Chem. 288, 20014-20033. doi: 10.1074/jbc. m112.438895

Naslund, T. I., Paquin-Proulx, D., Paredes, P. T., Vallhov, H., Sandberg, J. K., and Gabrielsson, S. (2014). Exosomes from breast milk inhibit HIV-1 infection of dendritic cells and subsequent viral transfer to CD4+ T cells. AIDS 28, 171-180. doi: 10.1097/qad.0000000000000159

Nguyen, D. G., Booth, A., Gould, S. J., and Hildreth, J. E. (2003). Evidence that HIV budding in primary macrophages occurs through the exosome release pathway. J. Biol. Chem. 278, 52347-52354. doi: 10.1074/jbc.m309009200

Ohno, S., Ishikawa, A., and Kuroda, M. (2013). Roles of exosomes and microvesicles in disease pathogenesis. Adv. Drug Deliv. Rev. 65, 398-401. doi: 10.1016/j.addr.2012.07.019

Park, I. W., and He, J. J. (2010). HIV-1 is budded from CD4+ T lymphocytes independently of exosomes. Virol. J. 7:234.

Patters, B. J., and Kumar, S. (2018). The role of exosomal transport of viral agents in persistent HIV pathogenesis. Retrovirology 15:79.

Pulliam, L., Sun, B., Mustapic, M., Chawla, S., and Kapogiannis, D. (2019). Plasma neuronal exosomes serve as biomarkers of cognitive impairment in HIV infection and Alzheimer's disease. J. Neurovirol. 25, 702-709. doi: 10.1007/ s13365-018-0695-4

Puzar Dominkus, P., Ferdin, J., Plemenitas, A., Peterlin, B. M., and Lenassi, M. (2017). Nef is secreted in exosomes from Nef.GFP-expressing and HIV-1infected human astrocytes. J. Neurovirol. 23, 713-724. doi: 10.1007/s13365017-0552-X

Qi, Y., Guo, L., Jiang, Y., Shi, Y., Sui, H., and Zhao, L. (2020). Brain delivery of quercetin-loaded exosomes improved cognitive function in $\mathrm{AD}$ mice by inhibiting phosphorylated tau-mediated neurofibrillary tangles. Drug Deliv. 27, 745-755. doi: 10.1080/10717544.2020.1762262

Qiu, Y., Ma, J., and Zeng, Y. (2018). Therapeutic potential of Anti-HIV RNAloaded exosomes. Biomed. Environ. Sci. 31, 215-226.

Qu, M., Lin, Q., Huang, L., Fu, Y., Wang, L., He, S., et al. (2018). Dopamine-loaded blood exosomes targeted to brain for better treatment of Parkinson's disease. J. Control Release 287, 156-166. doi: 10.1016/j.jconrel.2018.08.035

Rahimian, P., and He, J. J. (2016). HIV-1 Tat-shortened neurite outgrowth through regulation of microRNA-132 and its target gene expression. J. Neuroinflammation 13:247.

Raymond, A. D., Diaz, P., Chevelon, S., Agudelo, M., Yndart-Arias, A., Ding, H., et al. (2016). Microglia-derived HIV Nef+ exosome impairment of the bloodbrain barrier is treatable by nanomedicine-based delivery of Nef peptides. J. Neurovirol. 22, 129-139. doi: 10.1007/s13365-015-0397-0

Record, M., Subra, C., Silvente-Poirot, S., and Poirot, M. (2011). Exosomes as intercellular signalosomes and pharmacological effectors. Biochem. Pharmacol. 81, 1171-1182. doi: 10.1016/j.bcp.2011.02.011

Reynolds, J. L., and Mahajan, S. D. (2020). Transmigration of Tetraspanin 2 (Tspan2) siRNA via microglia derived exosomes across the blood brain barrier modifies the production of immune mediators by microglia cells. J. Neuroimmune Pharmacol. 15, 554-563. doi: 10.1007/s11481-019-09895-6

Reynolds, J. L., and Mahato, R. I. (2017). Nanomedicines for the treatment of CNS diseases. J. Neuroimmune Pharmacol. 12, 1-5. doi: 10.1007/s11481-017-9725-x

Rodriguez, M., Lapierre, J., Ojha, C. R., Estrada-Bueno, H., Dever, S. M., Gewirtz, D. A., et al. (2017). Importance of autophagy in mediating human immunodeficiency virus (HIV) and morphine-induced metabolic dysfunction and inflammation in human astrocytes. Viruses 9:201. doi: 10.3390/v9080201

Salarpour, S., Forootanfar, H., Pournamdari, M., Ahmadi-Zeidabadi, M., Esmaeeli, M., and Pardakhty, A. (2019). Paclitaxel incorporated exosomes derived from glioblastoma cells: comparative study of two loading techniques. Daru 27, 533-539. doi: 10.1007/s40199-019-00280-5

Sami Saribas, A., Cicalese, S., Ahooyi, T. M., Khalili, K., Amini, S., and Sariyer, I. K. (2017). HIV-1 Nef is released in extracellular vesicles derived from astrocytes: evidence for Nef-mediated neurotoxicity. Cell Death Dis. 8:e2542. doi: 10.1038/ cddis.2016.467

Schnell, G., Joseph, S., Spudich, S., and Swanstrom, R. (2001). HIV-1 replication in the central nervous system occurs in two distinct cell types. PLoS Pathog. 7:e1002286. doi: 10.1371/journal.ppat.1002286

Serlin, Y., Shelef, I., Knyazer, B., and Friedman, A. (2015). Anatomy and physiology of the blood-brain barrier. Semin. Cell. Dev. Biol. 38, 2-6.

Shelton, M. N., Huang, M. B., Ali, S. A., Powell, M. D., and Bond, V. C. (2012). Secretion modification region-derived peptide disrupts HIV-1 Nef's interaction with mortalin and blocks virus and Nef exosome release. J. Virol. 86, 406-419. doi: 10.1128/jvi.05720-11

Sillman, B., Woldstad, C., Mcmillan, J., and Gendelman, H. E. (2018). Neuropathogenesis of human immunodeficiency virus infection. Handb. Clin. Neurol. 152, 21-40. doi: 10.1016/b978-0-444-63849-6.00003-7

Smith-Vikos, T., and Slack, F. J. (2012). MicroRNAs and their roles in aging. J. Cell Sci. 125, 7-17. doi: 10.1242/jcs.099200

Sun, B., Fernandes, N., and Pulliam, L. (2019). Profile of neuronal exosomes in HIV cognitive impairment exposes sex differences. Aids 33, 1683-1692. doi: 10.1097/qad.0000000000002272

Tang, X., Lu, H., Dooner, M., Chapman, S., Quesenberry, P. J., and Ramratnam, B. (2018). Exosomal Tat protein activates latent HIV-1 in primary, resting CD4+ T lymphocytes. JCI Insight 3:e95676.

Tang, X., Lu, H., and Ramratnam, B. (2020). Neurotoxicity of HIV-1 Tat is attributed to its penetrating property. Sci. Rep. 10:14002.

Tang, Y., and Weidong, L. (2016). Differential roles of M1 and M2 microglia in neurodegenerative diseases. Mol. Neurobiol. 53, 1181-1194. doi: 10.1007/ s12035-014-9070-5

Valcour, V., Sithinamsuwan, P., Letendre, S., and Ances, B. (2011). Pathogenesis of HIV in the central nervous system. Curr. HIV/AIDS Rep. 8, 54-61. doi: 10.1007/s11904-010-0070-4

van Dommelen, S. M., Vader, P., Lakhal, S., Kooijmans, S. A., Van Solinge, W. W., Wood, M. J., et al. (2012). Microvesicles and exosomes: opportunities for cellderived membrane vesicles in drug delivery. J. Control Release 161, 635-644. doi: 10.1016/j.jconrel.2011.11.021

Venkat, P., Zacharek, A., Landschoot-Ward, J., Wang, F., Culmone, L., Chen, Z., et al. (2020). Exosomes derived from bone marrow mesenchymal stem cells harvested from type two diabetes rats promotes neurorestorative effects after stroke in type two diabetes rats. Exp. Neurol. 334:113456. doi: 10.1016/j. expneurol.2020.113456

Wang, C., Chen, L., Huang, Y., Li, K., Jinye, A., Fan, T., et al. (2019). Exosomedelivered TRPP2 siRNA inhibits the epithelial-mesenchymal transition of FaDu cells. Oncol. Lett. 17, 1953-1961.

Wang, C., Ravi, S., Garapati, Us, Das, M., Howell, M., Mallelamallela, J., et al. (2013). Multifunctional chitosan magnetic-graphene (CMG) nanoparticles: a theranostic platform for tumor-targeted codelivery of drugs, genes and MRI contrast agents. J. Mater. Chem. B Mater. Biol. Med. 1, 4396-4405. doi: 10.1039/ c3tb20452a

Wang, H., Sui, H., Zheng, Y., Jiang, Y., Shi, Y., Liang, J., et al. (2019). Curcuminprimed exosomes potently ameliorate cognitive function in $\mathrm{AD}$ mice by inhibiting hyperphosphorylation of the Tau protein through the AKT/GSK3beta pathway. Nanoscale 11, 7481-7496. doi: 10.1039/c9nr01255a

Wiley, R. D., and Gummuluru, S. (2006). Immature dendritic cell-derived exosomes can mediate HIV-1 trans infection. Proc. Natl. Acad. Sci. U.S.A. 103, 738-743. doi: 10.1073/pnas.0507995103

Xin, H., Li, Y., Liu, Z., Wang, X., Shang, X., Cui, Y., et al. (2013). MiR-133b promotes neural plasticity and functional recovery after treatment of stroke with multipotent mesenchymal stromal cells in rats via transfer of exosomeenriched extracellular particles. Stem Cells 31, 2737-2746. doi: 10.1002/stem. 1409 
Yang, J., Zhang, X., Chen, X., Wang, L., and Yang, G. (2017). Exosome Mediated Delivery of miR-124 Promotes Neurogenesis after Ischemia. Mol. Ther. Nucleic Acids 7, 278-287. doi: 10.1016/j.omtn.2017.04.010

Yang, L., Niu, F., Yao, H., Liao, K., Chen, X., Kook, Y., et al. (2018). Exosomal miR-9 released from HIV tat stimulated astrocytes mediates microglial migration. J. Neuroimmune Pharmacol. 13, 330-344. doi: 10.1007/s11481-0189779-4

Yang, T., Martin, P., Fogarty, B., Brown, A., Schurman, K., Phipps, R., et al. (2015). Exosome delivered anticancer drugs across the blood-brain barrier for brain cancer therapy in Danio rerio. Pharm Res. 32, 2003-2014. doi: 10.1007/s11095014-1593-y
Conflict of Interest: The authors declare that the research was conducted in the absence of any commercial or financial relationships that could be construed as a potential conflict of interest.

Copyright (c) 2021 Mahajan, Ordain, Kutscher, Karki and Reynolds. This is an openaccess article distributed under the terms of the Creative Commons Attribution License (CC BY). The use, distribution or reproduction in other forums is permitted, provided the original author(s) and the copyright owner(s) are credited and that the original publication in this journal is cited, in accordance with accepted academic practice. No use, distribution or reproduction is permitted which does not comply with these terms. 